\title{
Single-cell immune profiling of matched disease and recovery phase blood of COVID-19 patients reveals anti-LAG3 based immune checkpoint therapy as a promising option
}

\section{Qing Xiong}

Nanjing University Medical School

\section{Cheng Peng}

Nanjing University Medical School

Xiaomin Yan

Nanjing University Medical School

Lin Chen

Nanjing University Medical School

\section{Xueqi Yan}

Nanjing University Medical School

Beicheng Sun ( $\square$ sunbc@nju.edu.cn )

Nanjing University Medical School

Shiping Jiao ( $\nabla$ jiaoshp@tcrximmune.cn )

Nanjing University Medical School

\section{Research Article}

Keywords: COVID-19, SARS-CoV-2, Single cell sequencing, Exhausted T cell, Clonal expansion, Coinhibitory molecule

Posted Date: April 27th, 2020

DOl: https://doi.org/10.21203/rs.3.rs-24576/v1

License: (c) (i) This work is licensed under a Creative Commons Attribution 4.0 International License. Read Full License 


\section{Abstract}

We profiled the circulating immune cells in COVID-19 patients during the active disease and recovery phases via single cell RNA, TCR and BCR sequencing in order to elucidate the key anti-virus adaptive immune responses, and explore potential immunomodulatory therapeutic strategies. $T$ cell competence plays a dominant role in anti-SARS-CoV-2 immunity. Clonally expanded CD4 T effector cells averted CD8 $T$ cell exhaustion, and expansion of multiple CD8 T effector/memory clones was correlated with rapid virus clearance. In contrast, the patients lacking CD4 $\mathrm{T}_{\mathrm{EFF}}$ cell clonal expansion harbored exhausted CD8 $T_{\text {EFF }}$ cells co-expressing multiple co-inhibitory molecules. In addition, more than $90 \%$ of the exhausted cells expressed at least 2 and more than half of exhausted cells expressed at least 4 inhibitory molecules. Furthermore, co-expression of LAG3, Galectin-9, and SLAMF6 is the inhibitory molecule expression signature of exhausted T cell population in SARS-CoV-2 infection. Our findings indicate that LAG3-based immune checkpoint blockade is a promising strategy for treating COVID-19.

\section{Introduction}

The SARS-CoV-2 virus outbreak is now a global pandemic with over 2 million confirmed cases and 134,286 deaths across 185 countries and regions as of April 16th 2020 ${ }^{1}$. The occurrence, progression and subsequent clearance of viral infections largely depend on the ability of the host immune system to recognize, and respond specifically and effectively to the virus ${ }^{2}$. The innate immune response acts as a double-edged sword in viral infections; while the immune cells limit viral replication by producing antiviral cytokines like interferon (IFN)y and tumor necrosis factor (TNF)a, uncontrolled release of these factors can cause localized tissue damageas well as a systemic inflammatory response syndrome. The latter is the key immunopathological characteristic of COVID- $19^{3}$.

The adaptive immune response plays the key role in controlling virus infection. It has been proposed that antibodies produced by the B cells neutralize SARS-CoV-2 surface proteins that bind to host ACE2 receptors, and thus prevent the free virus particles from invading uninfected cells. In addition, in theory the antibody-coated free viruses trigger the complement cascade, and are eventually eliminated by the phagocytic innate immune cells. Consistent with this, preliminary results showed improvement in five critical COVID-19 patients treated with convalescent plasma presumably containing neutralizing antibodies ${ }^{4}$. Furthermore, several research groups are actively identifying SARS-CoV-2 neutralizing antibodies with single-cell sequencing of B cells. However, one previous study showed that the antiSARS-CoV antibodies promoted viral entry into uninfected cells because of antibody enhanced virus entry ${ }^{5}$. Recent study reported that an increased IgG response and a higher titre of total antibodies in COVID-19 patients were associated with severe disease and worse outcome ${ }^{6,7}$. These results were suggestive of possible antibody-dependent enhancement of SARS-CoV-2 infection. T cells are the primary effectors of cellular adaptive immune and indispensable for clearing the virus-infected cells, and limiting virus replication and spread. The cytotoxic $T$ lymphocytes (CTLs) are activated upon recognition of viral antigens by the T-cell receptor (TCR), and eliminate the infected cells by producing perforin granules, IFNY 
and granzymes ${ }^{8,9}$. Effective clearance of the infected cells is also necessary to mitigate the proinflammatory response of innate immune cells and protect the host tissues from secondary damage.

COVID-19 patients show significant changes in the absolute numbers as well as relative percentages of myeloid cells, $\mathrm{B}$ cells and $\mathrm{T}_{\text {cells }}{ }^{10}$, although the functional relevance vis-à-vis viral infection is still elusive. In addition, little is known regarding the T cell response specific to SARS-CoV-2. We profiled the immune cells in the blood samples from COVID-19 patients at the single cell level during both active disease and recovery phases, and analyzed the changes in the relative proportion and function of immune cells, the key cytokine origins, and T/B cell responses. The aim was to identify immune cell subset(s) responsible for virus control and the factors limiting anti-virus immunity, and propose new immunomodulatory approaches to treat SARS-CoV-2 infection.

\section{Results}

\section{Monocyte promotes anti-virus response via distinctly expressing TNFSF10 and TNFSF13B}

Two patients (designated PA0130 and PA0131) were admitted to the hospital with 5 or 6-day fever and dry cough. Neither patient had traveled to Hubei, but both had close contact with COVID-19 positive individuals travelling from Wuhan. Their throat swabs were positive for SARS-CoV-2 but negative for Influenza A/B virus, respiratory syncytial virus and adenovirus. Chest computed tomography (CT) further revealed progressive diffuse interstitial opacities and consolidation in the right lower lung fields (rapid progression stage) (Fig. 1A). Accordingly, both patients were diagnosed with moderate COVID-19 infection. PA0130 received lopinavir/ritonavir and low-dose prednisone after admission, and PA0131 additionally received acetylcysteine. The throat swab of PA0130 was negative for SARS-CoV-2 on day 21, while PA0131 showed viral clearance on day 13. Peripheral blood samples were collected from both patients on the day of admission and during the recovery phase, and subjected to single-cell RNA-seq, TCR-seq and BCR-seq (Fig. 1A and 1B).

Based on the single cell gene expression, the peripheral blood mononuclear cells (PBMCs) were clustered and annotated as CD 4 T cell, CD8 T cell, NK, NKT, B cell, plasma cell, classical/non-classical monocyte, megakaryocyte, dendritic cell, and plasmacytoid dendritic cell (Fig. 1C, 1D and Fig. S1). The relative proportion of monocytes increased in the disease phase as per the UMAP position in both PA0130 and PA0131 (Fig. 1C and 1D), indicating changes in its gene expression pattern and function during the infection. TNFSF10 and TNFSF13B were distinctly expressed in the classical and non-classical monocytes, and were upregulated during the infection (Fig. 1E and 1F). TNFSF10 induces apoptosis in virus-infected or transformed cells, and mediates the host immune surveillance against these cells ${ }^{11}$. TNFSF13B is linked to T cell-independent B cell activation ${ }^{12}$. Thus, the increased number of circulating monocytes in the COVID patients likely plays a role in viral clearance. The megakaryocyte subset showed a 7.9-fold increase in PA0130, but decreased significantly in PA0131. Nevertheless, megakaryocytes were the most abundant source of TGF $\beta$ in both patients (Fig. 1E and 1F). Virus-induced damage of lung tissues and pulmonary endothelial cells leads to platelet activation, aggregation and 
entrapment in the lungs, resulting in the formation of thrombi at the infected site that recruit more platelets and megakaryocytes ${ }^{2}$. Therefore, it is possible that megakaryocytes are also involved in COVID infection and immunomodulation. Finally, the proportion of CD8 T cells increased in PA0131 but not PA0130, while B cells showed the opposite trend (Fig. 1C and 1D).

\section{Clonal CD4 T cell expansion correlates low CD8 T cell exhaustion and multiple clonotypic expansions, and rapid virus clearance}

Since adaptive immune response is central to anti-virus immunity, we integrated the total gene expression data with the TCR/BCR-seq data to gain more insights into the clonal expansion of distinct $T$ and $B$ cell subsets. The clusters $0,2,3$ and 5 included the SELL+ CCR7+ TCF7+ naïve T cells (CD4 T NV$_{\text {) }}$ in PA0130. Cluster 6 and 9 consisted of a transient population between naïve and effector lymphocytes (CD8 $T_{N V}$ $\mathrm{EFF}$ ) which showed a decrease in the expression of lymphoid homing markers, and a corresponding increase in cytotoxic factors (PRF1, GZMB, GZMK, GZMH, or GNLY) and T cells activation markers (CCL5 and NKG7). Clusters 1 and 4 were defined as $T$ effector and memory cells (CD8 $T_{E F F}$ and $T_{E M}$ ) with low expression of homing markers, high expression of cytotoxic factors and IL7R+ KLRG1+ in the later. The high expression of CX3CR1 in cluster 1 indicated terminally- differentiated CD8 T cell effectors, while cluster 4 expressed high levels of IL7R and KLRG1, and intermediate levels of CD27 that are characteristic of long-lived memory cells (Fig. 2A and 2B).

An exhausted CD8 T cell subset expressing multiple co-inhibitory molecules was also observed in PA0130, and constituted $1.5 \%$ of all immune cells in the active disease phase before decreasing to $0.15 \%$ in the recovery phase (Fig. $2 \mathrm{C}$ ). Interestingly, the exhausted $\mathrm{T}$ cells showed the lymphoid homing

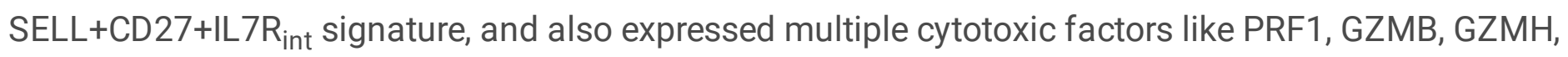
GZMK and GNLY. However, the high CASP3 expression indicated an exhausted state and short-lived fate. Consistent with this, TCR seq data pointed to non-clonal expansion of the exhausted CD8 T cell population. However, one CD8 $\mathrm{T}_{\mathrm{EFF}}$ clonotype in cluster 1 expanded at $6.23 \%$ compared to the entire population, and was predominant in the recovery stage as well (4.57\% on day 43$)$. We hypothesized that this particular clone (TRA: CALRLEYYGNKLVF; TRB: CASSPGQGTLAYEQYF) was SARS-CoV-2 specific, and was activated upon antigen stimulation. In support of our hypothesis, there was no clonal expansion of T cells (> 1\%) in two healthy blood samples (Fig. S2), indicating that the increased proportion of $\mathrm{T}$ cells in COVID-19 patients is stimulated by the SARS-CoV-2 antigens.

To further demonstrate T cell persistence in PA0130, we screened for clonotypes identical in the disease and recovery stages, and found them localized in the CD8 $T_{\text {EFF }}$ and CD8 $T_{E M}$ clusters (Fig. 2D). We did not observe CD4 T cell clonal expansion during the disease stage, although a very small percentage of CD4 $\mathrm{T}_{\mathrm{NV} \text {-EFF }}$ cells was identified (Fig. $2 \mathrm{C}$ ). Unlike the CD8 subsets, there were no overlapping CD4 T cell clonotypes between the disease and recovery stages, indicating that the CD4 T cells were short-lived and not persistent in circulation during the disease (Fig. 2D), which is supported by the CD4 $\mathrm{T}_{\mathrm{NV}}$ functional annotation as well (Fig. 2A). 
The T cells were similarly annotated in PA0131, and the CD4/CD8 naïve, effector and memory subsets were identified (Fig. 3A and 3B). Interestingly, PA0131 harbored expanded clonotypic CD4 T cells in circulation (Fig. 3C), and there were two major clonotypes with dramatically high expansion rates of $7.29 \%$ and $6.29 \%$ during the disease stage. Both clones persisted till day 47 and their expansion rates were $4.35 \%$ and $3.31 \%$ respectively. In addition, multiple CD8 T cell clonotypes were also detected in the disease phase that persisted during recovery (day 47), and no exhausted CD8 T cells were observed in this patient (Fig. 3A). The majority of clonally expanded T cells were CD $4 T_{E M}$ or $C D 8 T_{E M}$, and both populations expressed IL7R indicative of long-lived memory T cells (Fig. 3B).

A pseudotime trajectory was drawn to analyze the divergence between the expanded and non-expanded CD4 $\mathrm{T}_{\mathrm{EFF}}$ populations, using two clonotypes (1 and 2 ) with high expansion rates and the non-expanded cells in cluster 3. The pre-branch consisted of the non-expanded $C D 4 T_{E F F}$, while the two branches corresponded to the mixture of clonotypes 1 and 2. The non-expanded cells expressed high levels of SELL and ribosome-related genes but had lower cytokine gene expression compared to the expanded clonotypes, indicating that the former is an early-differentiation effector population and the latter is the major anti- viral effector. The difference between two branches on the trajectory is cell fate 1 branch showed enhanced cytotoxic capability with higher granule expressions than the other branch. Both clonotype 1 and 2 had distribution on the two branches, indicating each clonotype comprises heterogeneously differentiated effectors. High usage of TRBV7-9 or TRBV20-1 double confirmed that the populations come from clonal expansion. (Fig. 3E)

Interestingly, the analysis of BCR seq integrated with gene expression of both PA0130 and PA0131 revealed that there are limited clonotypes of $B$ cells with only slightly clonal expansions (Fig. S3A and 3B).

To summarize, the rapid virus clearance in PA0131 correlated to the expansion of multiple CD4 and CD8 T cell clonotypes, and lack of exhausted CD8 T cells. In contrast, PA0130 took longer to recover, and exhibited CD4 clonotypic expansion absence, only one predominant CD8 T cell clone as well as exhausted CD8 T cells expressing multiple co-inhibitory molecules. To validate the correlation between the host immunophenotype and viral clearance, we performed the same analysis on the blood samples of two additional patients with early (PA0133; 14 days) and late (PA0132; 29 days) viral clearance respectively (Table. 1). Similar to PA0130, PA0132 did not show any clonal expansion of CD4 T cells and only one expanded CD8 T cell clonotype (> 2\%; Fig. 4A), along with a short-lived CASP3+IL7R Iow exhausted CD8 T cell population expressing 6 co-inhibitory molecules (Fig. 4B). In contrast, PA0133 had multiple expanded CD4 and CD8 $T_{E M}$ clones in circulation, and lack of CD8 $T$ cell exhaustion similar to PA0131 (Fig. 4C and 4D). In addition, similar to those in PA0130 and PA0131, both PA0132 and PA0133 had limited B cell clonal expansions (Fig. S4).

\section{Exhausted CD8 $\mathrm{T}_{\mathrm{EFF}}$ cells in SARS-CoV-2 infection are featured by GZMA production and programmed by SUB1 and HMGB}


To further determine the accurate signature of exhausted CD8 T cells, we profiled the expression patterns of cytokines, chemokines, co-inhibitory/stimulatory molecules and transcription factors across the exhausted, non-expanded and expanded CD8 T cell subsets in PA0130 and PA0132. The genes differentially expressed across the 3 cell populations in more than $25 \%$ of the cells are shown in Figure 5A. Multiple co-inhibitory molecules were highly expressed only in the exhausted CD8 T cells, which incidentally also expressed the co-stimulatory molecules CD27 $7_{\text {int }}$ and CD28 $8_{\text {int }}$. In addition, GZMB and PRF1 rather than IFNy were identified as the major cytotoxic factors produced by the effector CD8 T cells against SARS-CoV-2 virus. We identified GZMA as the signature effector cytokine of the exhausted CD8 T cells, whereas CCL5 and CCL4 were the signature chemokines of the exhausted and expanded T cell subsets respectively. The highly expanded CD8 effector T cell clones expressed CX3CR1, which is indicative of terminal effector differentiation to the memory cells ${ }^{13,14}$. Studies on the mouse chronic virus infection such as LCMV and SIV models show that the balance between TBX21 and EOMES determines the T cell effector versus exhaustion fate. In the COVID-19 however, neither transcriptional factor was a determinant of T cell fate, and was expressed by a very small percentage of cells. In contrast, SUB1 and HMGB were the signature transcription factors of exhausted CD8 T cells, while PLEK and KLF2 programmed clonotypic CD8 T cells (Fig. 5A). However, the exact functional role of these transcriptional factors remains to be elucidated.

\section{Co-expression of LAG3, Galectin-9 and SLAMF6 is the predominant inhibitory receptor signature of exhausted CD8 $\mathrm{T}_{\text {EFF }}$ cells during SARS-CoV-2 infection}

Next, we analyzed the co-inhibitory molecule co-expression pattern in the exhausted $\mathrm{T}_{\text {EFF }}$ cells of PA0130 and PA0132. More than half of the exhausted CD8 $T_{\text {EFF }}$ cells in PA0130 and PA0132 co-expressed 4 molecules of LAG3, Gelectin-9 (LGALS9), SLAMF6, PD1 (PDCD1), CTLA4, TIM3 (HAVCR2), and TIGIT, $79.31 \%$ and $77.78 \%$ of the cells expressed at least 3 receptors, and $91.67 \%$ and $93.10 \%$ expressed at least 2 molecules in PA0130 and PA0132 respectively (Figure S5). Multiple co-inhibitory molecules coexpression parallels the severity of the exhaustion status. Consistent with this, the disease was more severe in PA0132 compared to PA0130. Notably, LAG3, Galectin-9 and SLAMF6 rather than PD1 and TIM3 are the predominant inhibitory molecules in the exhausted CD8 T cells of COVID-19 patients (Fig. 5B). In addition, $66.67 \%, 61.11 \%$ and $47.22 \%$ of the exhausted CD8 T cells co-expressed LAG3 with LGALS9 or SLAMF6 and their combination respectively in PA0130, whereas $58.62 \%, 46.55 \%$ and $34.48 \%$ of the cells were in PA0132. In chronic virus infection and cancer immunity, the signature of inhibitory molecule expression of exhausted T cells is featured by co-expression of PD1, TIM3 and LAG3 ${ }^{15-17}$. We further included the analysis of the exhausted T cells from non-HBV related or chronic HBV related hepatocellular carcinoma. Consistently, PD1, TIM3 and LAG3 or their combination are predominant co- inhibitory molecules co-expressed in both exhausted populations, while Galectin-9 and SLAMF6 showed low expression and less concurrence with LAG3 expression (Fig. 5C).

\section{Discussion}


SARS and COVID-19 are acute respiratory syndromes characterized by diffuse alveolar injury and pulmonary edema. However, the tissue damage is the result of a systemic inflammatory response to the virus-infected cells that are not rapidly cleared by the T cells, rather than virus-mediated cell lysis ${ }^{18,19}$. Furthermore, the immunosuppressive glucocorticoids given during severe inflammatory reactions can dampen adaptive immune response against the virus. Therefore, optimal activation of specific CTLs is crucial for blocking virus replication and dissemination, and inhibiting hyper-inflammation in COVID-19 patients.

T cell exhaustion is a dysfunctional state characterized by poor effector function due to sustained expression of inhibitory receptors, and is dependent on the duration and magnitude of antigenic stimulation ${ }^{20}$, CD4 T cell helper function, cytokines, and the expression levels of co-inhibitor/stimulatory receptors like PD1, TIM3 and CTLA4 ${ }^{21}$. It is well-established that CD4 T helper cells are essential for the expansion of CD8 T cells ${ }^{22}$. We found that the antigen-specific clonally expanded CD4 T cells drive CD8 T cell expansion during SARS-CoV-2 infection by averting the exhausted state, whereas the non- expanded CD4 $T$ cells are ineffectual.

The exhausted CD8 T cells identified in COVID-19 patients are distinct from the ones recognized in chronic virus infection and cancer. T cell subset with PD1 co-expressed with LAG3 and TIM3 are the dominant exhausted population during chronic HIV, HBV, HCV, SIV and LCMV infections and cancer ${ }^{21,23}$. However, PD1 + LAG3+ TIM3 + T cells constituted only $8-11 \%$ of the total exhausted T cell population in the acute SARS-CoV-2 infected patients. Instead, the majority of exhausted T cells co- expressed LAG3, Galectin-9 and SLAMF6 in COVID-19. TBX21 and EOMES are the primary transcription factors that determine the fate of PD1+ based exhausted T cells. In contrast, the LAG3+ LGASL9+ SLAMF6+ featured exhausted T cells in the COVID-19 patients were characterized by high expression of SUB1 and HMGB2. The biological relevance of both transcription factors in programming $T$ cell exhaustion remains to be elucidated.

Based on our findings, we hypothesize that immune checkpoint blockade (ICB) can enhance the adaptive immune response against COVID-19 by invigorating the exhausted CTLs. In fact, targeted blockade of PD1, TIGIT, TIM-3 or CTLA4 improved T cell responses against SIV, HIV and HBV infections in rhesus macaques and human cells ex vivo ${ }^{15,16,24,25}$. However, it may be the concern that the immune- related adverse events (irAEs) triggered by ICB, such as pneumonitis that is seen in $2.5-5 \%$ patients receiving antiPD1 therapy, may augment the systemic inflammation and lung tissue damage seen in SARS-CoV-2 infection. Nevertheless, irAEs are largely attributed to reduced T cell tolerance, whereas the excessive inflammation seen in SARS is the result of hyperactive innate immune cells and poor $\mathrm{T}$ cell response. For instance, PD-1 blockade in SIV-infected rhesus macaques enhanced T cell effector function and suppressed hyperimmune activation by downregulating type I IFN response ${ }^{26}$. Likewise, co-blockade of PD-1 and LAG-3 in the monkey SIV infection model synergistically improved the T cell response and reduced viral load ${ }^{21}$. Furthermore, irAEs usually develop 3-6 months after the initial dose of the immune 
checkpoint blocker ${ }^{27}$ and the median duration of hospitalization of COVID-19 patients is 12 days ${ }^{10}$, indicating an extremely low risk of potential irAEs overlapping with SARS-CoV-2-related inflammation.

Based on our findings, we surmise that ICB-mediated activation of virus-specific T cells would eliminate the infected cells, and effectively stymie the inflammatory response in COVID-19. The diverse HLA and TCR repertoires in the human population result in considerable heterogeneity in adaptive immune responses and clonotypic CD4 T cell expansion. Therefore, targeting one or more inhibitory molecules on CD8 T cells rather than manipulating virus-specific CD4 clonal expansion is more feasible for clinical applications. Antibodies to LAG3 are in clinical development and given their more favorable safety profiles may be more suitable agents to assess in SARS-CoV-2 infection. LAG3 blockade based immune checkpoint therapy can be potential therapeutic options to reinvigorate anti-SARS-CoV-2 T cells.

\section{Materials And Methods}

\section{Patients and ethics statement}

The study was conducted in accordance with the Declaration of Helsinki. Ethical approval was obtained from the Research Ethics Committee of Nanjing Drum Tower Hospital. All subjects gave their informed consent for sample collection and subsequent analyses.

\section{Subjects and clinical sample collection}

Four COVID-19 patients and 2 healthy donors were recruited from Nanjing Drum Tower Hospital from January to March, 2020. The demographic characteristics of the study population were listed in Table 1. All patients had cough and/or fever as the first symptom. SARS-CoV-2 was diagnosed on the basis of clinical symptoms, chest radiography, and sputum and throat swab viral PCR assays. The possibility of influenza A/B virus, respiratory syncytial virus and adenovirus infection were excluded at the time of enrollment.

\section{Hepatocellular carcinoma sample preparation}

For the isolation of TILs, tumor tissues were minced into small pieces and digested with collagenase Type II (100U/ml in HBSS containing calcium and magnesium) and DNase I $(20 \mu \mathrm{g} / \mathrm{ml})$ for $30 \mathrm{~min}$ at $37^{\circ} \mathrm{C}$, then prepared using the gentle MACS Dissociator (Miltenyi Biotec, Bergisch Gladbach, Germany) according to the manufacturer's protocol. Supernatants containing TILs were filtered through a $70 \mu \mathrm{m}$ cell strainer to obtain a single-cell suspension and then centrifuged at $800 \mathrm{~g}$ at $4^{\circ} \mathrm{C}$ for $30 \mathrm{~min}$ on a gradient consisting of $80 \%$ and $40 \%$ Percoll solutions (GE Healthcare, Uppsala, Sweden). The T cell fraction was recovered from the interface between the $80 \%$ and $40 \%$ Percoll layers. Tumor-infiltrating CD45+ $T$ cell subsets were stained with anti-CD 45 antibodies and sorted with a FACS Aria II cell sorter (BD Biosciences, CA, USA).

\section{Library preparation for $10 X$ Genomics single-cell $5^{\prime}$ gene expression and $V(D) J$ sequencing}


Single RNA-seq and V(D)J libraries were generated using the 10X Genomics Chromium Controller Instrument and Chromium single cell 5 ' library \& gel bead kit, along with the $V(D) J$ enrichment kit according to manufacturers' instructions. Briefly, 8000 peripheral blood mononuclear cells $>90 \%$ viability) were loaded on the controller to generate single-cell Gel Bead-In-Emulsions (GEMs). Reverse transcription and sample indexing were used to generate barcoded cDNA, followed by purification with Dyna Beads and PCR amplification. The amplified barcoded cDNA was used to construct 5 ' gene expression libraries, TCR and BCR enriched libraries. For 5 ' library construction, the amplified cDNA was fragmented, end repaired, A-tailed, sample indexed and double-sized selected with SPRI beads (average size, 450bp). For the $V(D) J$ library, human $T$ cell and $B$ cell $V(D) J$ sequences were enriched from the amplified cDNA followed by fragmentation, end repairing, A-tailing, sample indexing and double-sized selection with SPRI beads (average size, 600bp). The DNA quantification and fragment size distribution of the libraries were determined with Qubit dsDNA HS assay kit and Tape station. Pooled libraries were then sequenced on an Illumina high output sequencing platform, with $26 \mathrm{bp}$ on the first read and 98bp on the second read for gene expression analysis, and 150bp on the two reads for $V(D) J$ libraries.

\section{X library sequencing}

The scRNA libraries were sequenced on an Illumina Novaseq to a minimum sequencing depth of 50,000 reads per cell using reads lengths of $26 \mathrm{bp}$ read $1,8 \mathrm{bp}$ i 7 index, $151 \mathrm{bp}$ read 2 . The single-cell TCR and BCR libraries were sequenced an Illumina Novaseq to a minimum sequencing depth of 5,000 reads per cell using reads lengths of $151 \mathrm{bp}$ read $1,8 \mathrm{bp}$ i7 index, $151 \mathrm{bp}$ read 2 . For scRNA-seq, we obtained a total number of 2,889,964,940 reads with an average of 361,245,618 reads per sample and 49,016 reads per cell, and on average, 19,501 genes were detected per sample. For scTCR-seq, we obtained a total number of $269,471,477$ reads with an average of $33,683,935$ reads per sample and 11,167 reads per cell. For scBCR-seq, we obtained a total number of $238,810,972$ reads with an average of $29,851,372$ reads per sample and 30,426 reads per cell.

\section{Analyses of scRNA-seq data}

The scRNA-seq reads of each sample were independently aligned to the GRCh38 reference genome (10X Genomics, version 3.0.0) and quantified using cellranger count pipeline (10X Genomics, version 3.1.0) with default parameters. Filtered count matrix of features generated from cellranger count were then analyzed using Seurat (version 3.1.2) ${ }^{28}$. Genes expressed in less than 3 cells and cells with less than/equal to 200 or more than/equal to 5000 expressed genes or cells with more than/equal to $10 \%$ mitochondrial counts were discarded. We used the method developed by Yost et al. ${ }^{29}$ to remove batch effects between samples and cells related to $S$ and $\mathrm{G} 2 / \mathrm{M}$ cell cycle phases, a heat-shock gene expression signature, number of UMIs per cell, and mitochondrial RNA content when scaling the data. Variable genes were identified based on average of expression $>0.1$ and dispersion $>1$, and we used top 5000 variable genes for our analysis. To avoid clustering based on variable $\mathrm{V}(\mathrm{D}) \mathrm{J}$ transcripts, we removed variable TCR and immunoglobulin genes from the list of variable genes. We also removed genes in the $S$ and G2/M cell cycle signature and heat-shock signature to prevent clustering affected by these two factors. Cells 
were clustered using a shared nearest neighbor (SNN) modularity optimization-based clustering algorithm with the first 50 principal components and resolution set to 0.5 . Clusters were annotated manually based on the expression of known marker genes as indicated.

\section{Analyses of scTCR-seq and scBCR-seq}

The reads of scTCR-seq and scBCR-seq were independently aligned to the GRCh38 reference genome (10X Genomics, version 3.1.0) for each sample, and the clonotype analysis and TCR/BCR annotation were carried out using cellranger vdj pipeline (10X Genomics, version 3.1.0). For TCR clonotype analysis, we combined those clonotypes with same TRB/TRA CDR3 amino acid sequences since TRA/TRB CDR3 was probably missing due to low abundance.

\section{Trajectory analysis}

Trajectory analysis was performed by Monocle (version 2.12.0) ${ }^{30}$. Differential expression test of genes was performed between expanded and non-expanded CD4 $\mathrm{T}_{\mathrm{EFF}}$ populations using differential GeneTest function, and we used significant genes with q-value $<0.05$ as the ordering genes. We removed the effects of 5 covariates including size factor, percentage of mitochondrial counts, number of genes expressed, expression of the heat-shock gene signature, expression of $S$ and $\mathrm{G} 2 / \mathrm{M}$ cell cycle genes in the differential expression test. Dimension reduction was performed using DDRTree. To identify genes that contribute to cell fate differentiation, we performed differential expression test over pseudotime for each of the ordering genes, and we also regressed out the same 5 covariates as stated above in this test. We then used genes with q-value $<1 \mathrm{e}-40$ as significant genes for branched expression analysis (BEAM). We set num- clusters $=7$ and qval $=1 \mathrm{e}-4$ for generating the heatmap of branch genes .

\section{Declarations}

\section{Data availability}

All raw sequencing data of scRNA-seq, scTCR-seq, scBCR-seq will be deposited to GEO shortly, we will provide the accession number when it is available. All other relevant data are available from the corresponding authors upon reasonable request.

\section{Code availability}

All custom code used in this work is available from the corresponding authors upon reasonable request.

\section{Acknowledgements}

This work was supported by grants from the National Key Research and Development Program of China (2016YFC0905900 to B.S.), State Key Program of the National Natural Science Foundation (81930086 to B.S.), The Key Project by Medical Science and Technology Development Foundation, Nanjing Department 
of Health (ZKX2020001 to S.J.), Fundamental Research Funds for the Central Universities (021414380461 to B.S.). All authors claimed no conflict of interest.

\section{References}

1. Dong, E., Du, H. \& Gardner, L. An interactive web-based dashboard to track COVID-19 in real time. Lancet Infect Dis, doi:10.1016/S1473-3099(20)30120-1 (2020).

2. La Gruta, N. L. \& Turner, S. J. T cell mediated immunity to influenza: mechanisms of viral control. Trends Immuno/35, 396-402, doi:10.1016/j.it.2014.06.004 (2014).

3. Xu, Z. et al. Pathological findings of COVID-19 associated with acute respiratory distress syndrome. Lancet Respir Med, doi:10.1016/S2213-2600(20)30076-X (2020).

4. Shen, C. et al. Treatment of 5 Critically III Patients With COVID-19 With Convalescent Plasma. JAMA, doi:10.1001/jama.2020.4783 (2020).

5. Yang, Z. Y. et al. Evasion of antibody neutralization in emerging severe acute respiratory syndrome coronaviruses. Proc Natl Acad Sci U S A102, 797-801, doi:10.1073/pnas.0409065102 (2005).

6. Zhang, B. et al. Immune phenotyping based on neutrophil-to-lymphocyte ratio and IgG predicts disease severity and outcome for patients with COVID-19. medRxiv, 2020.2003.2012.20035048, doi:10.1101/2020.03.12.20035048 (2020).

7. Zhao, J. et al. Antibody responses to SARS-CoV-2 in patients of novel coronavirus disease 2019. Clinical Infectious Diseases, doi:10.1093/cid/ciaa344 (2020).

8. Kaech, S. M. \& Wherry, E. J. Heterogeneity and cell-fate decisions in effector and memory CD8+ T cell differentiation during viral infection. Immunity27, 393-405, doi:10.1016/j.immuni.2007.08.007 (2007).

9. Jiao, S. et al. Differences in Tumor Microenvironment Dictate T Helper Lineage Polarization and Response to Immune Checkpoint Therapy. Cel/179, 1177-1190 e1113, doi:10.1016/j.cell.2019.10.029 (2019).

10. Guan, W. J. et al. Clinical Characteristics of Coronavirus Disease 2019 in China. N Engl J Med, doi:10.1056/NEJMoa2002032 (2020).

11. Shin, G. C., Kang, H. S., Lee, A. R. \& Kim, K. H. Hepatitis B virus-triggered autophagy targets TNFRSF10B/death receptor 5 for degradation to limit TNFSF10/TRAIL response. Autophagy12, 2451-2466, doi:10.1080/15548627.2016.1239002 (2016).

12. Mackay, F. \& Schneider, P. Cracking the BAFF code. Nat Rev Immuno/9, 491-502, doi:10.1038/nri2572 (2009).

13. Bottcher, J. P. et al. Functional classification of memory CD8(+) T cells by CX3CR1 expression. Nat Commun6, 8306, doi:10.1038/ncomms9306 (2015).

14. Gerlach, C. et al. The Chemokine Receptor CX3CR1 Defines Three Antigen- Experienced CD8 T Cell Subsets with Distinct Roles in Immune Surveillance and Homeostasis. Immunity45, 1270-1284, doi:10.1016/j.immuni.2016.10.018 (2016). 
15. Wu, W. et al. Blockade of Tim-3 signaling restores the virus-specific CD8(+) T-cell response in patients with chronic hepatitis B. Eur J Immuno/42, 1180-1191, doi:10.1002/eji.201141852 (2012).

16. Velu, V. et al. Enhancing SIV-specific immunity in vivo by PD-1 blockade. Nature458, 206-210, doi:10.1038/nature07662 (2009).

17. thor Straten, P. et al. In situ T cell responses against melanoma comprise high numbers of locally expanded T cell clonotypes. J Immuno/163, 443-447 (1999).

18. Gu, J. et al. Multiple organ infection and the pathogenesis of SARS. J Exp Med202, 415- 424, doi:10.1084/jem.20050828 (2005).

19. Nicholls, J. M. et al. Lung pathology of fatal severe acute respiratory Lancet361, 1773-1778, doi:10.1016/s0140-6736(03)13413-7 (2003).

20. Mueller, S. N. \& Ahmed, R. High antigen levels are the cause of T cell exhaustion during chronic viral infection. Proc Natl Acad Sci U S A106, 8623-8628, doi:10.1073/pnas.0809818106 (2009).

21. Blackburn, S. D. et al. Coregulation of $\mathrm{CD} 8+\mathrm{T}$ cell exhaustion by multiple inhibitory receptors during chronic viral infection. Nat Immuno/10, 29-37, doi:10.1038/ni.1679 (2009).

22. Janssen, E. M. et al. CD4+ T cells are required for secondary expansion and memory in CD8+ T lymphocytes. Nature421, 852-856, doi:10.1038/nature01441 (2003).

23. Tirosh, I. et al. Dissecting the multicellular ecosystem of metastatic melanoma by single- cell RNAseq. Science352, 189-196, doi:10.1126/science.aad0501 (2016).

24. Chew, G. M. et al. TIGIT Marks Exhausted T Cells, Correlates with Disease Progression, and Serves as a Target for Immune Restoration in HIV and SIV Infection. PLoS Pathog12, e1005349, doi:10.1371/journal.ppat.1005349 (2016).

25. Schurich, A. et al. Role of the coinhibitory receptor cytotoxic T lymphocyte antigen-4 on apoptosisProne CD8 T cells in persistent hepatitis B virus infection. Hepatology53, 1494-1503, doi:10.1002/hep.24249 (2011).

26. Dyavar Shetty, R. et al. PD-1 blockade during chronic SIV infection reduces hyperimmune activation and microbial translocation in rhesus macaques. J Clin Invest122, 1712-1716, doi:10.1172/JCI60612 (2012).

27. Haanen, J. et al. Management of toxicities from immunotherapy: ESMO Clinical Practice Guidelines for diagnosis, treatment and follow-up. Ann Onco/28, iv119-iv142, doi:10.1093/annonc/mdx225 (2017).

28. Stuart, T. et al. Comprehensive Integration of Single-Cell Data. Cel/177, 1888-1902 e1821, doi:10.1016/j.cell.2019.05.031 (2019).

29. Yost, K. E. et al. Clonal replacement of tumor-specific T cells following PD-1 Nat Med25, 1251-1259, doi:10.1038/s41591-019-0522-3 (2019).

30. Trapnell, C. et al. The dynamics and regulators of cell fate decisions are revealed by pseudotemporal ordering of single cells. Nat Biotechno/32, 381-386, doi:10.1038/nbt.2859 (2014). 


\section{Tables}

Table 1. Clinical characteristics of COVID-19 patients PA0130 - 0134 and 2 health donors

\begin{tabular}{|c|c|c|c|c|c|c|}
\hline & PA0130 & PA0131 & PA0132 & PA0133 & $\begin{array}{l}\text { Health } \\
\text { donor } 1\end{array}$ & $\begin{array}{l}\text { Health } \\
\text { donor } 2\end{array}$ \\
\hline Age (Y) & 29 & 32 & 62 & 56 & 33 & 60 \\
\hline Gender & male & male & female & female & male & female \\
\hline First symptom & $\begin{array}{l}\text { Fever+ } \\
\text { cough }\end{array}$ & $\begin{array}{c}\text { Fever+ } \\
\text { cough }\end{array}$ & Fever & $\begin{array}{c}\text { Fever+ } \\
\text { cough }\end{array}$ & - & - \\
\hline SARS-CoV-2 & + & + & + & + & - & - \\
\hline Influenza $\mathbf{A}$ virus & - & - & - & - & - & - \\
\hline Influenza $B$ virus & - & - & - & - & - & - \\
\hline $\begin{array}{l}\text { Respiratory syncytial } \\
\text { virus }\end{array}$ & - & - & - & - & - & - \\
\hline Adenovirus & - & - & - & - & - & - \\
\hline CT finding & $\begin{array}{c}\text { Unilateral } \\
\text { pneumonia }\end{array}$ & $\begin{array}{c}\text { Unilateral } \\
\text { pneumonia }\end{array}$ & $\begin{array}{c}\text { Unilateral } \\
\text { pneumonia }\end{array}$ & $\begin{array}{c}\text { Unilateral } \\
\text { pneumonia }\end{array}$ & - & - \\
\hline Severity & moderate & moderate & severe & severe & - & - \\
\hline Moxifloxacin & + & + & - & - & - & - \\
\hline $\begin{array}{l}\text { Lopinavir / } \\
\text { Ritonavir }\end{array}$ & + & + & + & + & - & - \\
\hline Acetylcysteine & - & + & + & + & - & - \\
\hline Prednisone & - & + & + & + & - & - \\
\hline Hospitalization days & 22 & 14 & 32 & 16 & - & - \\
\hline virus clearance days & 21 & 13 & 29 & 14 & - & - \\
\hline
\end{tabular}

Figures 

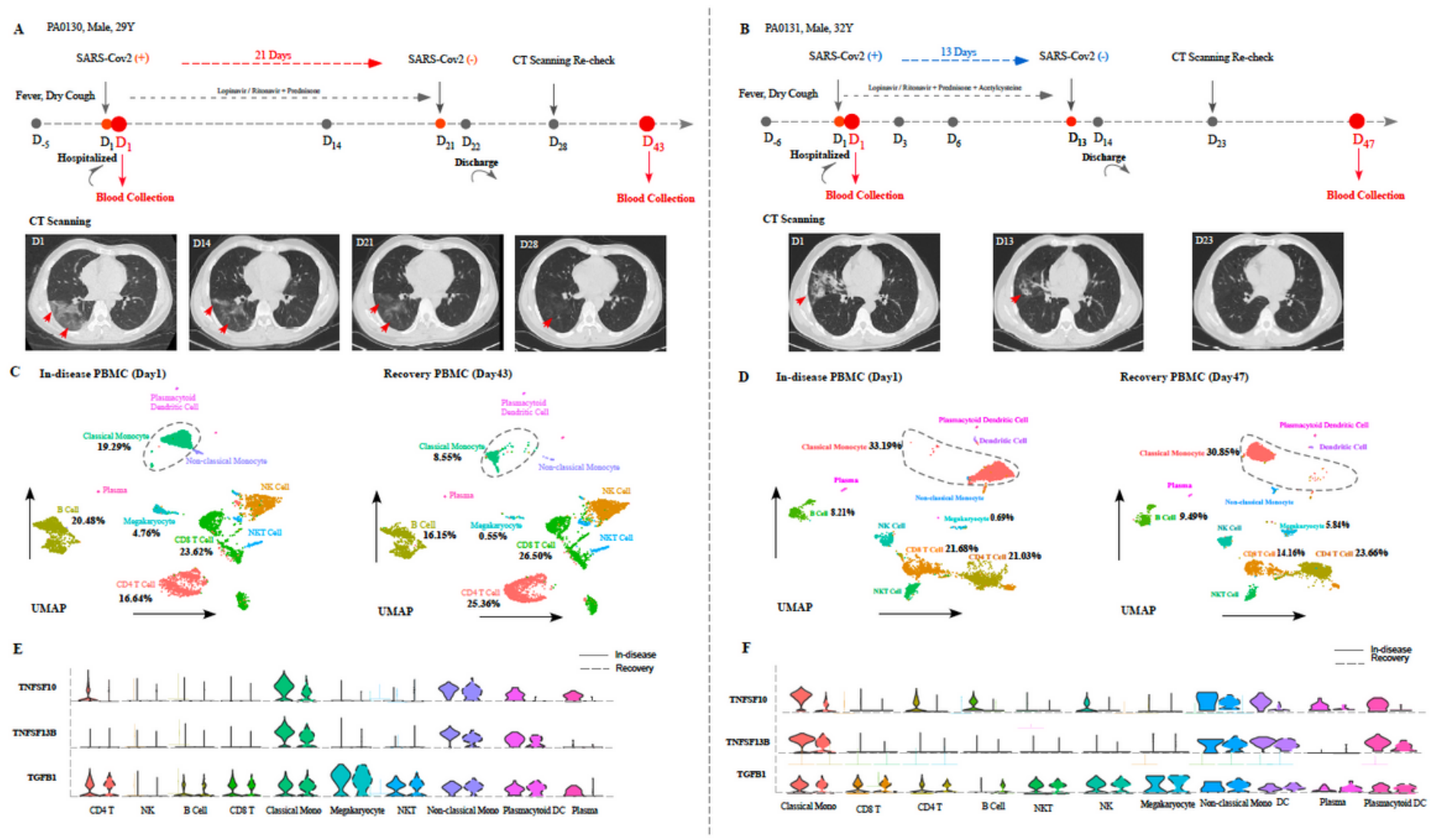

Figure 1

Clinical characteristics and peripheral immune cell subsets of PA0130 and PA0131. (A and B). The timeline of PA0130 and PA0131 disease course, blood sample collections, treatment and clinical testing/imaging. D-5 or D- 6 indicates 5 or 6 days before the time of hospitalized. (C and D) UMAP plots to illustrate the total circulating immune cell subsets in the patients. The percentage is calculated by each cell subset number/ total immune cells. Each immune cell subset is annotated based on the core markers listed in Figure S1. (E and F) Violin plots to show TNFSF10, TNFSF13B or TGFß as the distinct cytokines of monocytes or megakaryocytes. Solid and Dash lines indicated the same cell type during disease and recovery phase. 


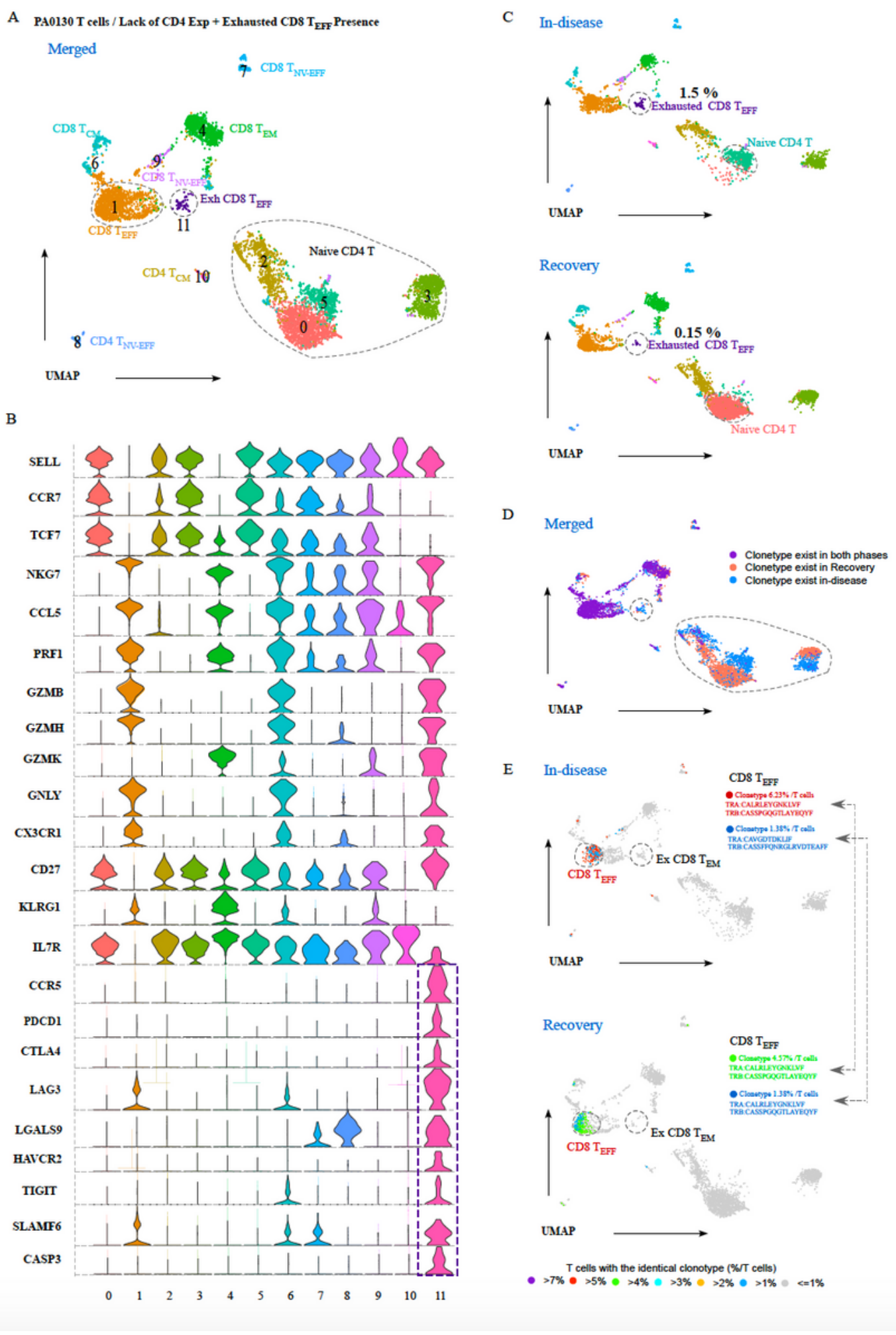

\section{Figure 2}

CD4 T cell clonal expansion is absent in PA0130 and associated with the presence of exhausted CD8 T cells. (A and B) T cells of PA0130 during disease and recovery phases were merged and clustered. T cell subsets were annotated based on the core maker expressions plotted in (B). TNV-EFF, TEFF, TEM, TCM indicated naïve-effector, effector, effector-memory, central memory T cells. (C) In order to see the percentage change of exhausted $T$ cell population, $T$ cells were split to separated plots based on the time- 
in-disease and recovery. (D) Purple color indicated T cells with the clonotypes identified in both diseaseand recovery- samples. Blue and golden color indicated the ones only found in disease- and recoveryphase, respectively. (E) TCR seq analysis was integrated with gene expression data. The T cells with identical TCR CDR3 sequences were identified as one clonotype. Clonotypes with T cells more than $1 \%$ of the total T cells were considered as significant clonal expansion. Aliases for genes: SELL - CD62L; IL7R CD127; PDCD1 - PD1; LGALS9 - Galectin-9; HAVCR2 - TIM3. Figure S2 and S3A are related to Figure 2.
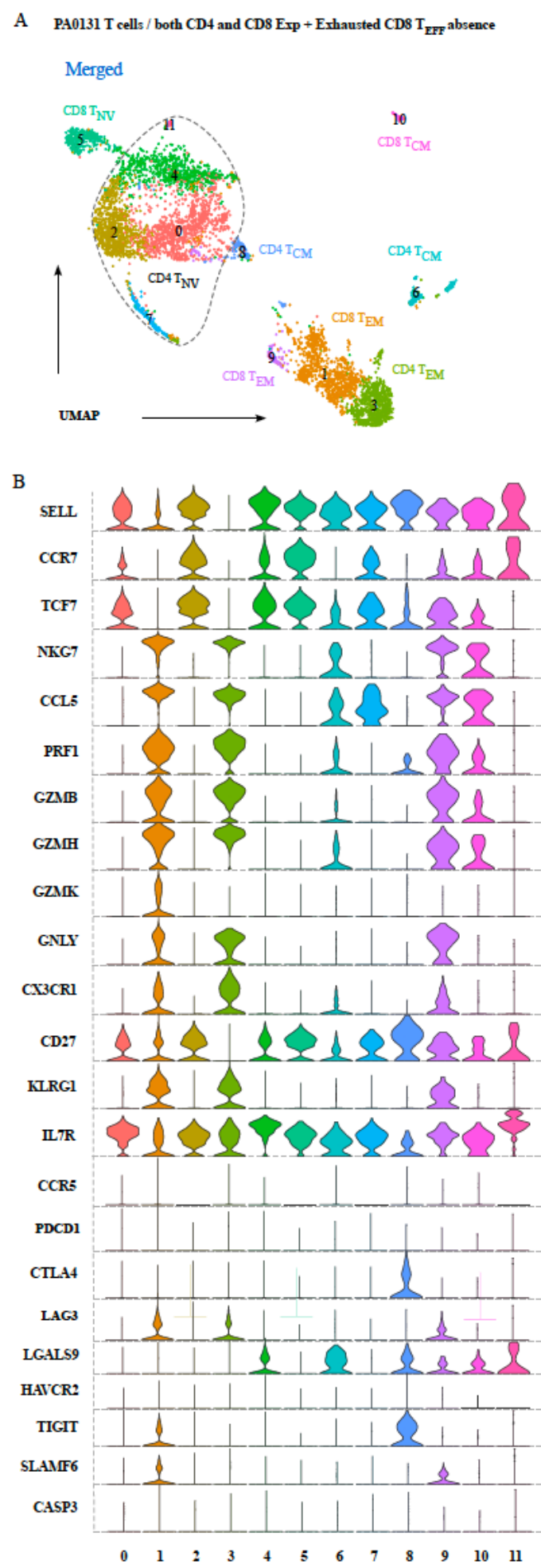

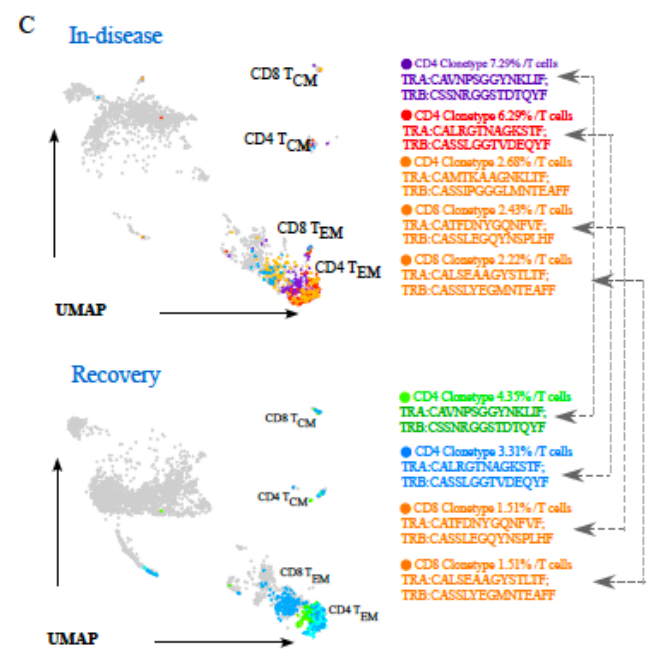

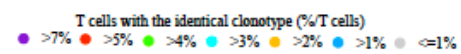

D PA0131 T Cells Merged

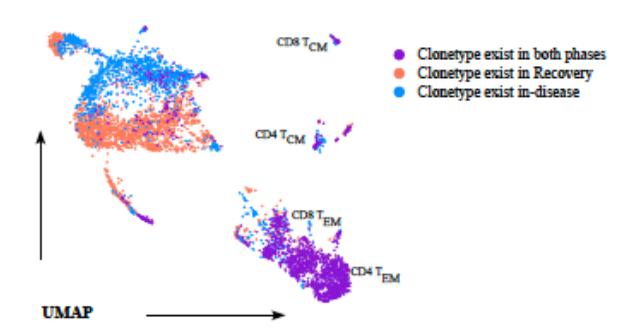

$\mathrm{E}$
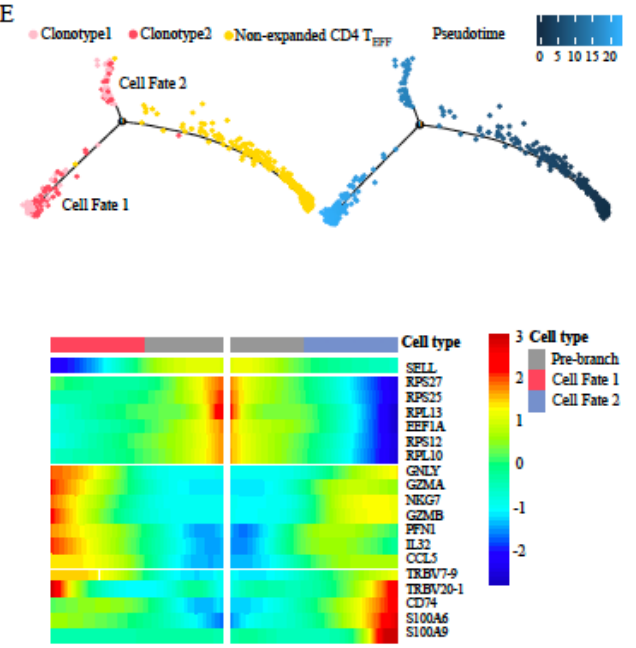
Circulating T cells in PA0131 comprised both expanded CD4 and CD8 clonotypes but no exhausted T cells. (A and B) T cells of PA0131 during disease and recovery phase were merged and clustered. T cell subsets were annotated as that in PA0130. Exhausted T cells were not found. (C) Clonal expansion analysis based on TCR seq identified multiple highly expanded CD4 and CD 8 clonotypes. Both CD4 and CD8 clonotypic $T$ cells were persistent and existed in peripheral blood after 47 days. (D) Shared clonotype analysis further supported the long-live fate of the expanded T cells. (E) Monocle2 analysis of the CD4 T cells consisted of expanded clonotype 1 and 2 CD 4 T cells and non-expanded CD4 T effectors in cluster 3. The beam heatmap indicated the key markers that distinguish the different fates of the total analyzed cells. Figure S3B is related to Figure 3.
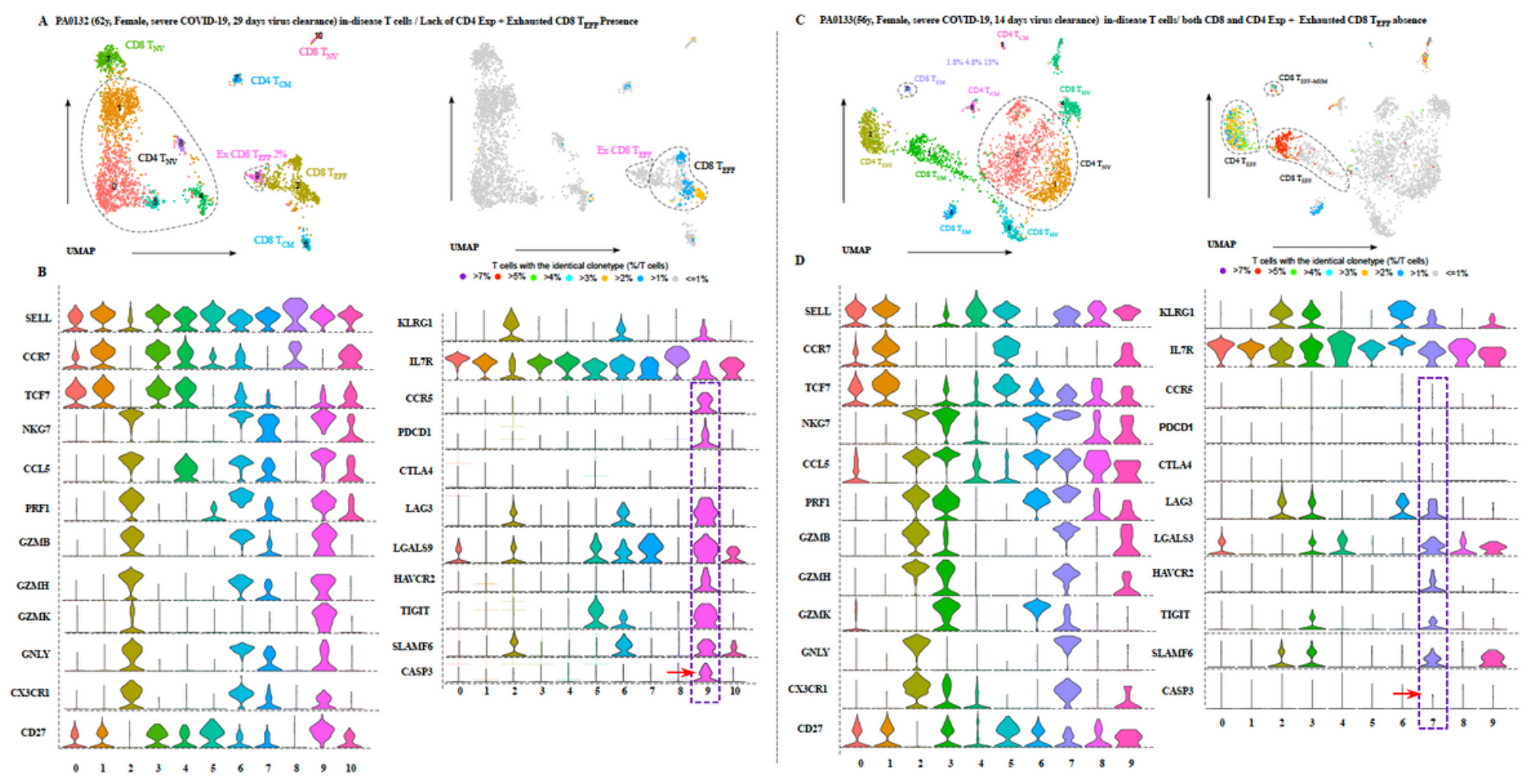

\section{Figure 4}

Clonal expansion of CD4 T cells in PA0133 correlates with CD8 T cell exhaustion and virus clearance. (A and B) The T cell subsets and their clonal expansion information of PA0132 and PA0133. The key clinical characterizes are indicated on the plots but with the details in Table 1. ( $C$ and D) Key markers to define $T$ cell subsets in the blood of each patient. Figure S4 is related to Figure 4. 


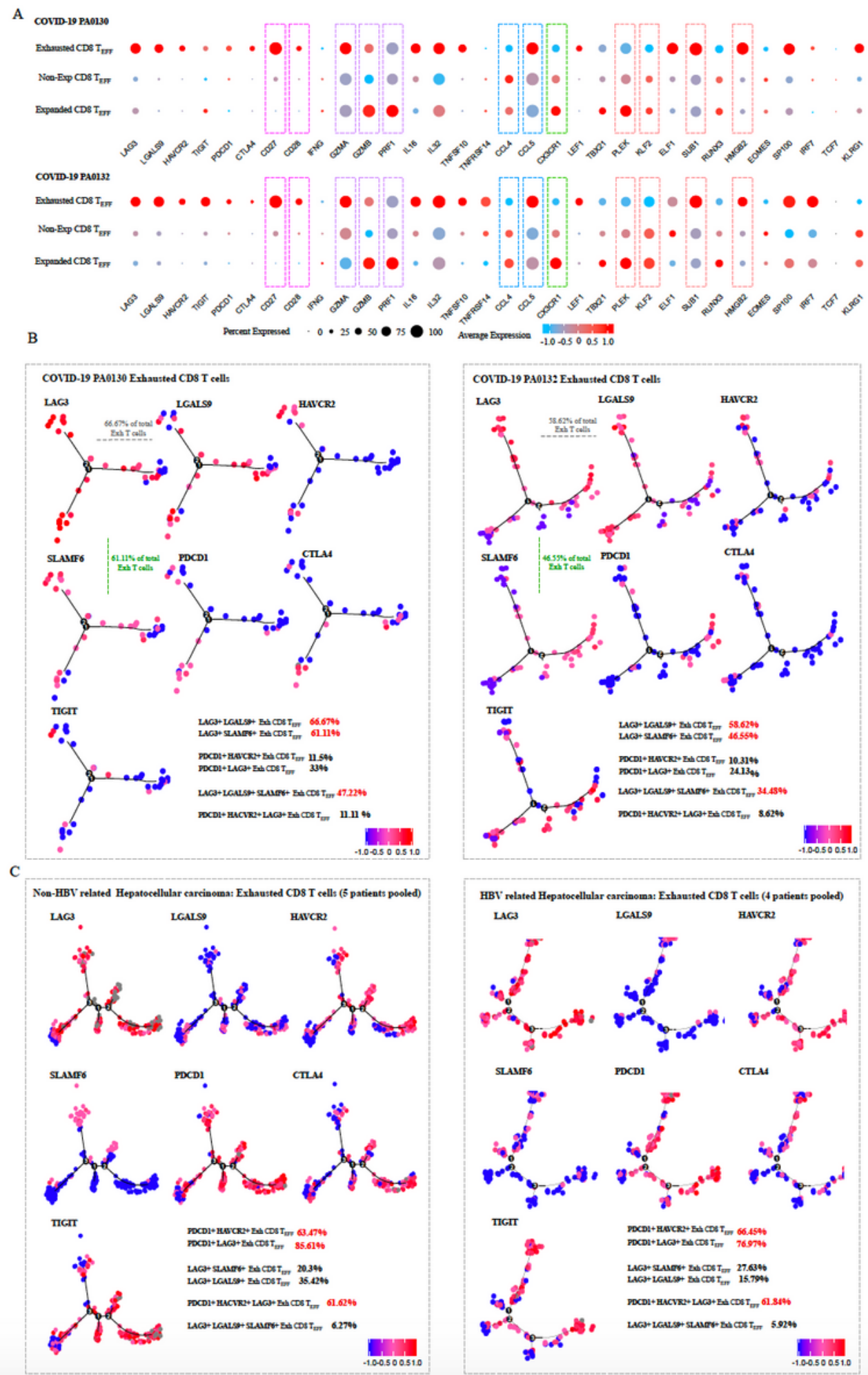

\section{Figure 5}

Exhausted CD8 T cells in SARS-CoV-2 infection are distinct from the ones recognized in chronic virus infection and cancer. (A) The expanded clonotypic CD8 T cells, non-expanded TEFF cells in cluster 1 and exhausted CD8 TEFF (cluster 11) in PA0130 were set as the 3 subpopulations to compare their expressions of cytokines, chemokines, co-inhibitory/ stimulatory molecules and transcriptional factors. The genes differentially expressed across the 3 sub-populations in more than $25 \%$ of the cells are shown 
with bubble plots. In the analysis of PA0132, the 3 subsets - exhausted, non-expanded and expanded CD8 TEFF- were cluster 9 shown in Figure 4A, non-expanded TEFF and expanded TEFF in cluster 2, relatively. (B) Co-inhibitory molecule co-expression signature (LAG3, CTLA4, TIGIT, SLAMF6, PDCD1 - PD1; LGALS9 Galectin-9; HAVCR2 - TIM3) was analyzed in each individual cell of exhausted CD8 T cells (cluster 11) in PA0130 and of the ones (cluster 9) in PA0132. The red or blue color labeled cell indicated the one with high or low expression of the marker. The percentage indicated cell number (co-expression of 2 or 3 molecules) / total exhausted cells. (C) Co-expression analysis of inhibitory molecules in exhausted CD8 T cells pooled from 5 non-HBV or 4 HBV related hepatocellular carcinoma patients. Patient's tumor infiltrated immune cells were sorted and through single cell $V(D) J$ immune profiling. After cell subset annotated by core markers, the data of exhausted T cell population were identified and extracted.

\section{Supplementary Files}

This is a list of supplementary files associated with this preprint. Click to download.

- Supplementarylnformation.pdf 